\title{
Perioperative Management of Cleft Palate Repair in a Patient with Williams Syndrome: A Case Report
}

\author{
Balaji Asegaonkar ${ }^{1 *}$, Jiten J. Kulkarni², Rajgopal J. Totla², Pulkeshi Kulkarni ${ }^{3}$, Shilpa Asegaonkar ${ }^{4}$, \\ Santosh Totla ${ }^{5}$ \\ ${ }^{1}$ Department of Anesthesiology, Mahatma Gandhi Mission Medical College, Aurangabad, India; ${ }^{2}$ Department of Plastic and Pediatric \\ Surgery, Mahatma Gandhi Mission Medical College, Aurangabad, India; ${ }^{3}$ Consultant Anesthesiologist, Aurangabad, India; ${ }^{4}$ De- \\ partment of Biochemistry, Goverment Medical College, Aurangabad, India; ${ }^{5}$ Consultant Ophthalmologist, Aurangabad, India. \\ Email: ${ }^{*}$ b_asegaonkar@yahoo.com
}

Received October $20^{\text {th }}, 2012$; revised November $24^{\text {th }}, 2012$; accepted December $24^{\text {th }}, 2012$

\begin{abstract}
The Background: Williams syndrome is a neurodevelopmental multisystem genetic disorder characterized by dysmorphic features and a wide range of congenital cardiac, renal, musculoskeletal anomalies. Presence of cleft palate in these patients is an unusual condition. More than 22 cases of sudden cardiac death reported in literature especially during anesthetic management in the patients of Williams syndrome. So these patients present major perioperative challenges to anesthesiologist. The authors report successful anesthetic care for repair of cleft palate in presence of uncorrected patent ductus arteriosus (PDA) and mild supraventricular aortic stenosis (SVAS) in a 3.5-year-old female child diagnosed with Williams syndrome. Case report: Diagnosed case of Williams syndrome with characteristic facial dysmorphic features, SVAS and rare malformation of PDA, was scheduled for cleft palate repair. After keen preoperative assessment, general anesthesia was administered for the procedure with continuous monitoring for vital parameters. Because of dysmorphic features, difficulty was faced during intubation. Otherwise intraoperative procedure was uneventful. Postoperative analgesia was managed with intravenous paracetamol. Conclusion: Being a multisystem disorder, each patient of WS requires meticulous preoperative evaluation and high level of intraoperative and postoperative continuous monitoring regardless of any surgical procedure. Surgical correction of cleft palate in such patients demands very high anesthetic and surgical skills as both share common airway. Present case report highlights the significance of aggressive perioperative management in WS which can result in successful outcome.
\end{abstract}

Keywords: Williams Syndrome; PDA; SVAS; Cleft Palate Repair; Perioperative Management

\section{Introduction}

Williams syndrome (WS) or Williams-Beuren syndrome, a sporadic genetic neurodevelopmental disorder was first described by Williams, Barratt-Boyes and Lowe in 1961. [1] It is associated with microdeletion of about 25 - 30 genes spanning 1.5 megabases in region of q 11.23 of long arm on chromosome 7 . This region includes gene for the protein elastin. Its prevalence is estimated to be 1 in 7500 to 1 in 20,000 live births [2].

Clinical features of WS include facial dysmorphism characterized by a distinct elfin facial appearance with low nasal bridge, mental retardation with unusual language skills, visiospatial deficits, hyperoscialibility, stellate iris pattern, transient hypercalcemia, genitourinary manifestations, and distinctive behavioral and emotional traits. $50 \%$ to $80 \%$ cases have cardiovascular anomalies due to defective elastin gene. Cardiac lesions reported in Williams syndrome are supravalvular or subvalvular aor-

\footnotetext{
${ }^{*}$ Corresponding author.
}

tic stenosis (72\%), valvar or peripheral pulmonary stenosis (39\%), hypertension (17\%), mitral valve prolapse $(15 \%)$, coarctation of aorta $(4 \%)$ of the cases. Other rare cardiac congenital defects are ASD, PDA, Tetralogy of Fallot, coronary artery stenosis and endocardial cushion defect [3].

In literature sudden cardiac deaths have been reported in the patients of Williams syndrome and over half of the sudden cardiac deaths were associated with anesthesia for these patients during cardiac catheterization and other surgical procedures [4]. This suggests significance of careful vigilance during these procedures. More than 22 sudden deaths have been reported in patients with Williams syndrome and out of which many of them occurred during anesthetic management [5]. Because of multiple system involvement and congenital anomalies perioperative anesthetic management of these patients is very challenging. We are presenting a report about the perioperative management for repair of cleft palate in diagnosed case of Williams syndrome, although cleft pal- 
ate is not a usual manifestation of WS. As per our knowledge, after extensive search in medical literature, no such case has been reported in Indian population regarding anesthetic management of cleft palate repair in WS. We also reviewed past case reports related to anesthetic care of WS and discussed their perioperative management. Informed consent from patient's relative was obtained for publishing case report.

\section{Case Report}

A 3.5-year-old girl child weighing $10 \mathrm{Kg}$ with William syndrome was referred to our centre for cleft palate repair. She was diagnosed case of Williams syndrome by neuropaediatrician at the age of 1 year when she was evaluated for failure to thrive and systolic murmur. After this her main complain was inability to eat solid food and unclear voice. She was delivered during 32-week of gestation and her birth weight was 2 kilograms. Physical examination revealed characteristic dysmorphic facial features of elfin like face, flat nasal bridge, upturned nose, wide mouth, clenoductaly and long philtrum (Figure 1). She had borderline intelligence and history of delayed milestones. There was no history of seizures, thyroid dysfunction and any surgical procedure in past.

On physical examination her vital signs were found to be within normal limits for her age. Heart rate was 120/minute and blood pressure 90/40 mmHg. Clinically she was pale and hemoglobin was $6.3 \mathrm{gm} \%$ which was corrected to $11.1 \mathrm{gm} \%$ with two packed cell volume transfusions with diuretics over a period of four days. Other biochemical parameters were within normal limit. We specifically investigated her for serum calcium and thyroid function tests. During preoperative evaluation she was afebrile with pulse 110 /minute, respiratory rate 24/minute, and $\mathrm{SpO}_{2} 99 \%$ on pulse oxymetry. Chest radiograph showed moderate cardiomegaly. On auscultation ejection systolic murmur was present in aortic area. Electrocardiogram was without any ST-T changes or any hypertrophy changes. She was referred to cardiologist for detail cardiological examination. Echocardiography evaluation in cardiology clinic revealed mild supravalvular aortic stenosis (SVAS) with pressure gradient of $25 \mathrm{~mm}$ Hg with small patent ductus arteriosus (PDA) but without pulmonary hypertension. Cardiologist advised device closure for PDA and cardiac catheterization but patient's relatives were reluctant and insisted for cleft palate repair earlier. Potential risk during anesthesia and surgery was explained to parents in detail, still they insisted for surgery as the patient was unable to eat solid food and speak properly because of cleft palate. Written high risk consent was obtained from parents and patient posted for surgery of repair of cleft palate under general anesthesia.

Morning dose of tablet Ranitidine $10 \mathrm{mg}$ was given

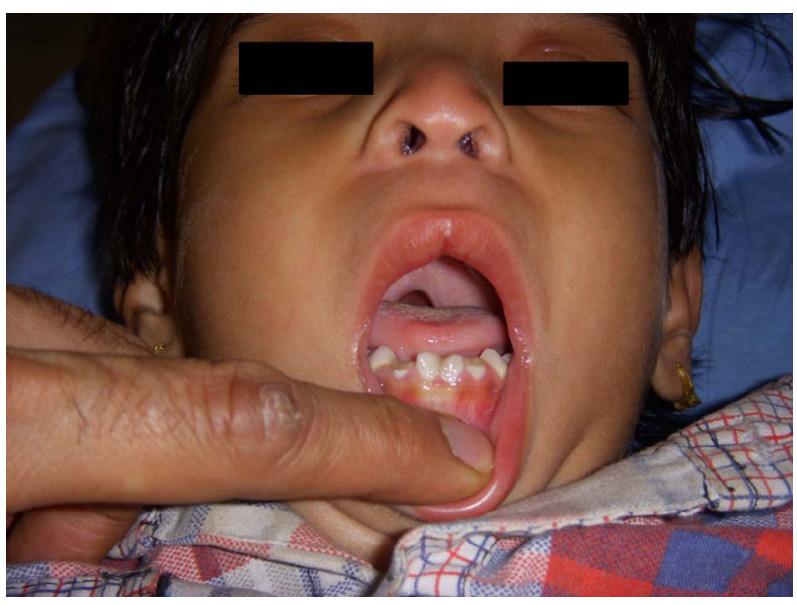

Figure 1. Facial dysmorphic features.

with sip of water. The child was kept nil by mouth for six hours, intravenous access taken with $22 \mathrm{G}$ angiocath after applying local anesthetic cream (Prilax). Half an hour before induction, bolus of antibiotic and injection Tranexamic acid $10 \mathrm{mg} / \mathrm{Kg}$ was administered slowly intravenously. Patient sedated with Midazolam $0.1 \mathrm{mg} / \mathrm{Kg}$ and Fentanyl $12 \mathrm{mcg} / \mathrm{kg}$. Preoxygenation started and as she became calm, continuous monitoring with electrocardiography, noninvasive blood pressure, $\mathrm{SpO}_{2}$, peripheral $\mathrm{O}_{2}$ saturation and end tidal $\mathrm{CO}_{2}$ started. After 5 minutes of preoxygenation patient was induced with Propofol $2 \mathrm{mg} / \mathrm{Kg}$, Ketamin $0.5 \mathrm{mg} / \mathrm{kg}$ and Atracurium 0.5 $\mathrm{mg} / \mathrm{Kg}$. After three minutes patient was intubated with RAE 4.5 plain endotracheal tube. Air entry on both sides confirmed, then tube was fixed with proper sticking and throat pack kept. During intubation visualization of vocal cords was difficult due to characteristic facial dysmorphic features and required more tracheal pressure externally. Patient handed over to surgeon. Cleft palate defect was closed by correcting position of muscles of soft palate and reconstruction of muscle sling. Anesthesia maintained on oxygen, nitrous, isoflurone and intermittent atracurium.

During intraoperative phase there was one episode of tachycardia which was settled with additional dose of Fentanyl 5 microgram and deepening plane of anesthesia with Isoflurone. All vital and hemodynamic parameters were stable and continuously monitored during intraoperative period and patient did not showed signs of awareness. Surgery lasted for two hours and after confirming haemostasis, throat packs removed. Effect of relaxant reversed with Neostigmin $0.04 \mathrm{mg} / \mathrm{Kg}$ and Glycopyrolate $0.01 \mathrm{mcg} / \mathrm{Kg}$. After gaining adequate power, patient was extubated, oxygenation continued and patient observed for 15 minutes in operation theatre. With continuous monitoring patient was shifted to pediatric intensive care unit where she was observed for 24 hours and pain was managed with intravenous paracetamol 30 
$\mathrm{mg} / \mathrm{Kg}$. On fifth day she was discharged without any adverse event.

\section{Discussion}

The authors report successful anesthetic care for repair of cleft palate in presence of uncorrected PDA and mild SVAS in a 3.5-year-old female child diagnosed with Williams syndrome.

Williams syndrome, a multisystem disorder with multiple congenital heart anomalies poses several anesthetic problems perioperatively. These patients require sedation or general anesthesia for various surgical procedures and cardiac catheterization. In past decade case reports related to perioperative management in Williams syndrome with congenital heart disease revealed adverse events on induction of anesthesia. At least 22 cases of sudden cardiac death have been documented in literature due to myocardial infarction, decreased cardiac output, and ventricular arrhythmias [5-7]. So meticulous care at all time including preoperative evaluation, intraoperative and postoperative period is very essential regardless of the procedure.

We discussed our experience about perioperative anesthetic care for cleft palate repair in WS and also previous case studies in such patients. Cleft palate is not a clinical feature reported in medical literature as a part of WS. This might be rare entity in these patients [8] (Figure 2). There is a limited data about the anesthetic care of patients with WS for cleft palate. Monfred reported sudden cardiac death after completion of tonsillectomy during periextubation period [9]. Sevtap et al. reported successful anesthetic care for inguinal hernia repair in patient with WS [10].

In patients of Williams syndrome absence of elastin gene leads to muscle weakness resulting in joint laxity which is concerned with anesthetic care. Laxity of joints with craniofacial dysmorphic features cause difficulty during tracheal intubation and airway management [11].

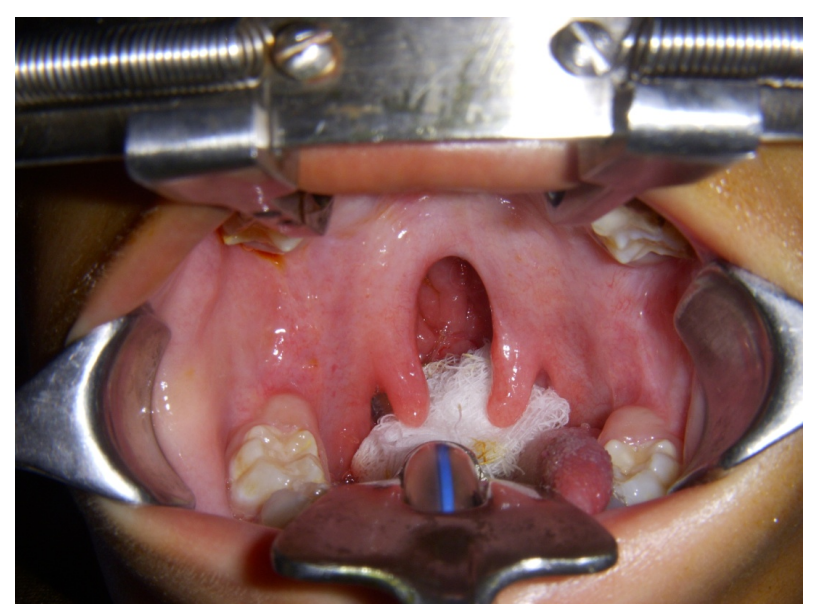

Figure 2. Cleft palate appearance after intubation.
There might be difficulty even for mask ventilation and these patients may require smaller endotracheal tube than expected for the age. Also there are high chances of postoperative stridor due to laryngeal edema [5]. About 40\% of the patients have anatomical anomalies of renal system. Multiple renal problems in these patients are renovascular hypertension, hypercalcemia induced nephrocalcinosis, structural renal abnormalities like renal aplasia, horseshoe kidney and renal cyst $[12,13]$. Anesthetist has to administer titrated dosages of nondepolarising blockade with nerve stimulators due to prolonged neuromuscular blockage [14]. So thorough preoperative assessment including cardiological examination, renal function, airway anatomy, metabolic parameters, joint mobility, and dental check up, level of cognitive function regardless of procedure plays crucial role to plan and monitor the patient. This help to anticipate potential risk for safety intervention. Thorough explanation of potential risk associated and benefits of the procedure should be discussed with parents.

As far as cardiovascular problems are concerned, supravalvular aortic stenosis and coronary artery disease (CAD) are associated most commonly in the patients with Williams Syndrome [15]. Initially it was thought to may be due to endocrine disturbances and hypercalcemia. But subsequently it was proved to be due to deletion of elastin gene that causes it. Accelerated process of atherosclerosis can lead to CAD which to some extent can be attributed to hypercalcemia and hypothyroidism [16]. Bird et al. reported most of the fatalities in these patients occur either immediately after induction or completion of anesthesia. Also they commented that severity of SVAS is not predictor of sudden death [6]. Our patient had mild supravalvular aortic stenosis which is a common anomaly in WS, but presence of associated PDA was a new and rare finding in WS. Ideally patient should have undergone device closure for PDA and cardiac catheterization before cleft palate repair. But patient's relatives were reluctant for it and hence cleft palate repair planned earlier with due risk.

We specifically investigated our patient for hypercalcemia and thyroid function. If hypercalcemia is present one must look for renal and gastrointestinal pathologies like nephrocalcinosis, constipation, failure to thrive, abdominal colic and vomiting. Also hypothyroidism is a common finding associated with this disorder which raises perioperative risk considerably. So preoperative screening for thyroid function tests is must in cases with Williams syndrome. Presence of hypothyroidism may lead to depressed myocardial function, anemia, decreased plasma volume, hypoglycemia, and hyponatremia and decreased drug metabolism in liver and poorly controlled temperature regulation [17]. But in our case fortunately thyroid function tests were within normal limit. 
Surgical correction of cleft palate in such patients demands very high anesthetic and surgical skills as both share common airway. Being a multisystem disorder, each patient of WS requires meticulous preoperative evaluation and high level of intraoperative and postoperative continuous monitoring regardless of any surgical procedure. Present case report highlights the significance of aggressive perioperative management in WS which can result in successful outcome.

\section{REFERENCES}

[1] J. C. Williams, B. G. Barratt-Boyes and J. B. Lowe, "Supraventricular Aortic Stenosis,” Circulation, Vol. 24, 1961, pp. 1311-1318. doi:10.1161/01.CIR.24.6.1311

[2] P. Stromme, P. G. Bjornstad and K. Ramstad, "Prevalence Estimation of Williams Syndrome,” Journal of Child Neurology, Vol. 17, No. 4, 2002, pp. 269-271. doi:10.1177/088307380201700406

[3] P. P. Wang and N. J. Blum, "Williams Syndrome: Disorders of Development and Learning,” pp. 283-298.

[4] P. Gupta, J. D. Dobias, S. Goyal, M. D. Miller, E. Melendez, N. Navaski, et al., "Sudden Cardiac Death under Anesthesia in Pediatric Patient with Williams Syndrome: A Case Report and Review of Literature," Annals of Cardiac Anesthesiology, Vol. 13, No. 1, 2010, pp. 44-48.

[5] J. Medley, P. Russo and J. D. Tobias, "Perioperative Care of the Patient with Williams Syndrome," Pediatric Anesthesia, Vol. 15, No. 3, 2005, pp. 243-247. doi:10.1111/j.1460-9592.2004.01567.x

[6] L. M. Bird, G. F. Billman, R. V. Lacro, et al., "Sudden Death in Williams Syndrome: Report of 10 Cases," The Journal of Pediatrics, Vol. 129, No. 6, 1996, pp. 926-931. doi:10.1016/S0022-3476(96)70042-2

[7] P. E. Horowitz, S. Akhtar, J. A. Wulff, et al., "Coronary Artery Disease and Anesthesia Related Death in Children with Williams Syndrome,” Journal of Cardiothoracic and Vascular Anesthesia, Vol. 6, No. 6, 2002, pp. 739-741. doi:10.1053/jcan.2002.128407

[8] F. Blanco-Davila and J. A. Olveda-Rodriguez, "Cleft palate in a patient with Williams' Syndrome,” The Journal of Craniofacial Surgery, Vol. 12, No. 2, 2002, pp. 145147.

[9] A. Monfared and A. Messner, "Death Following Tonsillectomy in a Child with Williams Syndrome,” International Journal of Pediatric Otorhinolaryngology, Vol. 70, No. 6, 2006, pp. 1133-1135. doi:10.1016/j.ijporl.2005.11.009

[10] S. H. Sahin, A. Colak and I. Gunday, "Anesthesia Management of a Patient with Williams Syndrome,” The Internet Journal of Anesthesiology, Vol. 14, No. 1, 2007.

[11] A. Lashkari, A. Smith and J. Graham, "Williams-Beuren Syndrome: An Update and Review for the Primary Physician,” Clinical Pediatrics, Vol. 38, No. 4, 1999, pp. 189208. doi:10.1177/000992289903800401

[12] R. Pankau, C. J. Partch, M. Winter, et al., "Incidence and Spectrum of Renal Abnormalities in Williams-Beuren syndrome," American Journal of Medical Genetics, Vol. 63, No. 1, 1996, pp. 301-304. doi:10.1002/(SICI)1096-8628(19960503)63:1<301::AIDAJMG49>3.0.CO;2-P

[13] B. R. Pober, R. V. Lacro, C. Rice, et al., "Renal Findings in 40 Individuals with Williams Syndrome,” American Journal of Medical Genetics, Vol. 46, No. 3, 1993, pp. 271-274. doi:10.1002/ajmg.1320460306

[14] M. G. Butler, B. G. Hayes, M. M. Hathaway, et al., "Specific Genetic Diseases at Risk for Sedation/Anesthesia Complications,” Anesthesia Analgesia, Vol. 91, No. 4, 2000, pp. 837-855. doi:10.1097/00000539-200010000-00014

[15] B. Mohan and C. M. Mittal, "Supravalvular Aortic Stenosis in Williams Syndrome," Annals of Paediatric Cardiology, Vol. 4, No. 2, 2011, pp. 213-214.

[16] J. Audrzejowski and J. Mundy, "Anaesthesia for MRI Angiography in a Patient with Williams Syndrome," Anaesthesia, Vol. 55, No. 1, 2000, pp. 97-98. doi:10.1046/j.1365-2044.2000.01254.x

[17] V. Cammareri, G. Vignati, N. Gianfranco, et al., "Thyroid Hemiagenesis and Elevated Thyrotropin Levels in a Child with Williams Syndrome,” American Journal of Medical Genetics, Vol. 85, No. 5, 1999, pp. 491-494. doi:10.1002/(SICI)1096-8628(19990827)85:5<491::AIDAJMG11>3.0.CO;2-Z 